\title{
Quasisynchronization in Quorum Sensing Systems with Parameter Mismatches
}

\author{
Jianbao Zhang, ${ }^{1}$ Wenyin Zhang, ${ }^{2}$ Zhongjun Ma, ${ }^{3,4}$ and Jing Yu ${ }^{1}$ \\ ${ }^{1}$ School of Science, Hangzhou Dianzi University, Hangzhou 310018, China \\ ${ }^{2}$ School of Informatics, Linyi University, Linyi 276005, China \\ ${ }^{3}$ School of Mathematics and Computing Science, Guilin University of Electronic Technology, Guilin 541004, China \\ ${ }^{4}$ Guangxi Key Laboratory of Trusted Software, Guilin University of Electronic Technology, Guilin 541004, China \\ Correspondence should be addressed to Zhongjun Ma; mzj1234402@163.com
}

Received 2 April 2014; Revised 1 July 2014; Accepted 1 July 2014; Published 16 July 2014

Academic Editor: Chuangxia Huang

Copyright (c) 2014 Jianbao Zhang et al. This is an open access article distributed under the Creative Commons Attribution License, which permits unrestricted use, distribution, and reproduction in any medium, provided the original work is properly cited.

\begin{abstract}
The paper investigates quasisynchronization in a communication system, which consists of cells communicating through quorum sensing. With the help of Lyapunov function method and Lure system approach, some sufficient conditions for quasisynchronization are presented, and a bound on the synchronization errors is derived. The obtained theoretical results show that the synchronization quality is influenced by two parameters detrimentally: the error bound depends almost linearly on the mismatches between cells and depends sensitively on the diffusion rates of the signals inward the cell membrane. Numerical experiments are carried out to verify the theoretical results.
\end{abstract}

\section{Introduction}

In the past decades, increasing interest has been shown to study the dynamics of coupled oscillator networks, which describes many complex systems in the field of nature and science. Due to the couplings among these oscillators, different types of synchronization can be realized in such systems.

In particular, a coupled oscillator network is called to be quasisynchronized, or weak synchronized, if the synchronization errors will be in some neighborhood of zero but will not tend to zero eventually. In other words, quasisynchronization means that the dynamical trajectories of each cell are similar but different from each other [1,2]. By Lyapunov function method and a differential inequality method, two coupled identical oscillators with parameter mismatches were quasisynchronized via periodically intermittent control in [3]. Similar results were also obtained in a discontinuous master-response system with parameter mismatches [4]. It has also been shown that two coupled delayed oscillators with parameter mismatches can be lag quasisynchronized, and the error level is estimated by applying a generalized Halanary inequality and matrix measure [5].
To the best of our knowledge, most of researches on synchronization focused on two oscillators coupled directly [6-8]. And there are very few researches focused on the system composed of several oscillators coupled indirectly. However, many biological systems coupled indirectly can also exhibit synchronization such as a global cellular response. For an example of such mechanisms achieving synchronization, the unicellular bacteria are highly coupled through chemical signaling molecules. This process, termed quorum sensing [9-11], allows bacterial populations to exchange intercellular signals with their neighbor cells to coordinate gene expression and integrates cells to realize synchronization effectively. That is to say, each bacterium is connected with all the other bacteria through a mean field and coordinated precisely. As the result, all the bacteria form a "microsociety," behaving synchronously and exhibiting various collective dynamics. In the past decade, synchronization induced by the intercell signaling mechanism has been widely investigated. For instance, synchronization induced by quorum sensing has been studied in networks composed by genetic relaxation oscillators [12], limit-cycle oscillators [13], and synthetic gene oscillators [14]. Another research has shown that a noisy community of such genetic oscillators can self-synchronize 
in a robust way and lead to a substantially global rhythmicity [15].

Due to the biological diversity, there are usually some parameter mismatches between the coupled oscillators in biological systems. Therefore, complete synchronization is hard to be achieved. Instead, quasisynchronization, which implies a state of synchronization with an error level, is more common in the biological systems. Motivated by the complexity and similarity of biological organisms and the potential applications, the paper studies quasisynchronization in quorum sensing systems with parameter mismatches. Through a new method different from many previous researches [3-5], the bound on the synchronization errors is estimated with help of Lur'e system, linear matrix inequalities, and Lyapunov function [16-19]. Both the theoretical results and the numerical simulations indicate that the synchronization errors stay in a neighborhood of zero, increase roughly linearly with the mismatches between individual cells, and depend sensitively on the diffusion rate of the signals inward the cell membrane.

The rest of the paper is organized as follows. In Section 2, we give some sufficient conditions for quasisynchronization in quorum sensing systems with parameter mismatches. The bound of the synchronization errors is also estimated. In Section 3, numerical examples are carried out to verify the theoretical results.

\section{Main Results}

2.1. Quorum Sensing Systems with Mismatches. Quorum sensing is a cell concentration dependent phenomenon in bacteria and fungi, which is mediated by small, diffusible signaling molecules that accumulate in the extracellular environment [9]. In such a multicell system, the individual oscillator in each cell is a network with three genes, $a, b$, and $c$, the products of which inhibit the transcription of each other in a cyclic way. The gene $c$ expresses protein $C$, which inhibits transcription of the gene $a$. The product of $a$ inhibits transcription of the gene $b$, the protein product $B$ of which in turn inhibits expression of $c$, completing the cycle. These bacteria exhibit cell-to-cell communication through a mechanism that makes use of two proteins, the first one of which (LuxI) synthesizes a small molecule known as an autoinducer (AI), which can diffuse freely through the cell membrane. The principle of the phenomenon is that when a single bacterium releases autoinducers (AIs) into the environment, their concentration is too low to be detected. However, when sufficient bacteria are present, AI concentrations reach a threshold level that allows the bacteria to sense a critical cell mass and to activate target genes [9]. When a second protein (LuxR) binds to this molecule, the resulting complex activates transcription of various genes, including some coding for light-producing enzymes. The scheme of the network is shown in Figure 1. For further details, one is referred to previous articles [9-11].

Before we carry out the dynamics model for the $\mathrm{N}$ cell system described by differential equations, we make the following declaration throughout the paper. Let $R^{n}$ be the $n$ dimensional Euclidean space, $R^{n \times m}$ the set of all $n \times m$ real

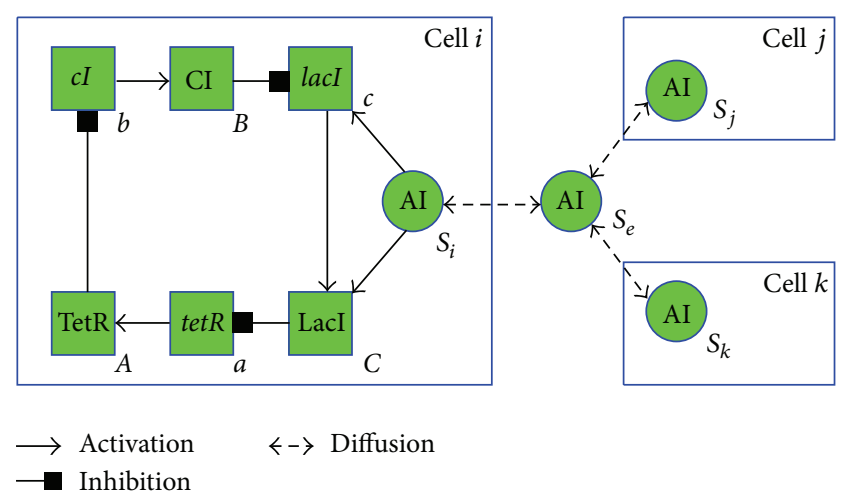

Figure 1: Scheme of the repressilator network coupled through signaling molecules, termed quorum sensing. The synchronization scheme of quorum sensing is based on the diffusion of a small molecule (autoinducer AI) to and from the cells.

matrices, $A^{\top}$ the transpose of a square matrix $A$, and $\|\cdot\|$ the usual $L_{2}$ norm of a vector or the usual spectral norm of a square matrix. The notation $M>0(<0)$ is used to define a real symmetric positive (negative) definite matrix. If not explicitly stated, matrices are assumed to have compatible dimensions.

Then the dynamics model for the $N$-cell system is built as follows:

$$
\begin{gathered}
\dot{a}_{i}=-d_{1 i}(t) a_{i}+\frac{\alpha_{6 i}(t)}{\mu_{6}+C_{i}^{m}}, \quad \dot{A}_{i}=-d_{4 i}(t) A_{i}+\beta_{1 i}(t) a_{i}, \\
\dot{b}_{i}=-d_{2 i}(t) b_{i}+\frac{\alpha_{4 i}(t)}{\mu_{4}+A_{i}^{m}}, \quad \dot{B}_{i}=-d_{5 i}(t) B_{i}+\beta_{2 i}(t) b_{i}, \\
\dot{c}_{i}=-d_{3 i}(t) c_{i}+\frac{\alpha_{5 i}(t)}{\mu_{5}+B_{i}^{m}}+\frac{\alpha_{7 i}(t) S_{i}}{\mu_{7}+S_{i}}, \\
\dot{C}_{i}=-d_{6 i}(t) C_{i}+\beta_{3 i}(t) c_{i}, \\
\dot{S}_{i}=-d_{7 i}(t) S_{i}+\beta_{4 i}(t) A_{i}-\eta\left(S_{i}-S_{e}\right), \\
\dot{S}_{e}=-d_{e}(t) S_{e}+\eta_{e}(t) \sum_{j=1}^{N} \frac{S_{j}-S_{e}}{N},
\end{gathered}
$$

where $i=1,2, \ldots, N ; a_{i}, b_{i}$, and $c_{i}$ are the concentrations of $m R N A$ transcribed from genes $a, b$, and $c$ in cell $i$, respectively; $A_{i}, B_{i}$, and $C_{i}$ are the concentrations of the corresponding proteins, respectively; $S_{i}$ and $S_{e}$ are concentrations of AI inside each cell and in the environment, respectively. The parameters $d_{j i}(t)(j=1, \ldots, 7)$ and $d_{e}(t)$ are the dimensionless degradation rates of the chemical molecules in cell $i ; \alpha_{j i}(t)(j=4,5,6)$ are the dimensionless transcription rates in the absence of repressor; $\alpha_{7 i}(t)$ is the maximal contribution to the gene $c$ transcription in the presence of saturating amounts of $\mathrm{AI} ; \beta_{j i}(t)(j=1,2,3)$ are the translation rates of the proteins from the $m R N A s ; \beta_{4 i}(t)$ is the synthesis rate of AI; $m=4$ is the Hill coefficient; $\eta(t)$ and $\eta_{e}(t)$ measure the diffusion rate of AI inward and outward the cell membrane. 
We suppose that all the parameters mentioned above are time-varying in the vicinity of certain constants, which indicates that all the cells are similar but different from each other. For convenience, we decompose these parameters into two parts: a constant part that determines the values of the parameters and a time-varying part representing the parameter mismatches. For example, let the parameter $d_{j i}(t)=d_{j}+\delta d_{j i}(t),(j=1, \ldots, 7)$, which implies that the parameter $d_{j i}(t)$ is time-varying around the constant $d_{j}$ with the parameter mismatches $\delta d_{j i}(t),(j=1, \ldots, 7)$.

Denoting $x_{i}=\left(a_{i}, b_{i}, c_{i}, A_{i}, B_{i}, C_{i}, S_{i}\right)^{\top}, i=1, \ldots, N$, and $x_{e}=\left(0,0,0,0,0,0, S_{e}\right)^{\top}$ for convenience, then muiticell system (1) can be rewritten as follows:

$$
\begin{gathered}
\dot{x}_{i}=-\left(d+\delta d_{i}(t)\right) x_{i}+\left(\alpha+\delta \alpha_{i}(t)\right) f\left(x_{i}\right) \\
-\left(\gamma+\delta \gamma_{i}(t)\right)\left(g\left(x_{i}\right)-e_{n}\right)+\eta\left(x_{e}-I_{n} x_{i}\right), \\
\dot{x}_{e}=-d_{e}(t) x_{e}+\eta_{e}(t) \sum_{j=1}^{N} \frac{\left(I_{n} x_{j}-x_{e}\right)}{n},
\end{gathered}
$$

where $i=1,2, \ldots, N ; d=\operatorname{diag}\left(d_{1}, d_{2}, d_{3}, d_{4}, d_{5}, d_{6}, d_{7}\right)+\beta$, $\beta \in R^{n \times n}$ with elements equal to zero except $\beta_{41}=\beta_{1}$, $\beta_{52}=\beta_{2}, \beta_{63}=\beta_{3}$, and $\beta_{74}=\beta_{4} ; \alpha \in R^{n \times n}$ with elements equal to zero except $\alpha_{16}=\alpha_{6}, \alpha_{24}=\alpha_{4}$, and $\alpha_{35}=\alpha_{5}$; $\gamma \in R^{n \times n}$ with elements equal to zero except $\gamma_{37}=\alpha_{7}$; $I_{n}=\operatorname{diag}(0,0,0,0,0,0,1) ; e_{n}=(0,0,0,0,0,0,1)^{\top} ;$ and

$$
\begin{gathered}
g\left(x_{i}\right)=\left(0,0,0,0,0,0, \frac{\mu_{7}}{\mu_{7}+S_{i}}\right)^{\top}, \\
f\left(x_{i}\right)=\left(0,0,0, \frac{1}{\mu_{4}+A_{i}^{m}}, \frac{1}{\mu_{5}+B_{i}^{m}}, \frac{1}{\mu_{6}+C_{i}^{m}}, 0\right)^{\top} .
\end{gathered}
$$

The matrices $\delta d_{i}(t), \delta \alpha_{i}(t)$, and $\delta \gamma_{i}(t)$ describe the mismatches of the parameters $d_{i}(t), \alpha_{i}(t)$, and $\gamma_{i}(t)$. Note the dimension of individual cells $n=7$ for multicell system (1). In fact, system (2) can describe the general model of quorum sensing mechanism, where the vector components of vector functions $f(\cdot)$ and $g(\cdot)$ are increasing functions and $I_{n}$ describes which components are coupled with the environment. Thus, the results obtained in the paper are also valid for any general multicell system based on quorum sensing.

2.2. Sufficient Conditions for Quasisynchronization. Since the cells in realistic organisms are similar but different from each other, one can suppose that the mismatch matrices $\delta d_{i}(t)$, $\delta \alpha_{i}(t)$, and $\delta \gamma_{i}(t)$ are bounded as follows:

$$
\left\|\delta d_{i}(t)\right\| \leq \varepsilon_{1}, \quad\left\|\delta \alpha_{i}(t)\right\| \leq \varepsilon_{2}, \quad\left\|\delta \gamma_{i}(t)\right\| \leq \varepsilon_{3} .
$$

Noticing that the concentrations of chemical molecules $x_{i}(t)$ are bounded and $f(\cdot)$ and $g(\cdot)$ are monotonic functions of $x_{i}(t)$, we can conclude that

$$
\left\|x_{i}(t)\right\| \leq \delta_{1}, \quad\left\|f\left(x_{i}(t)\right)\right\| \leq \delta_{2}, \quad\left\|g\left(x_{i}(t)\right)\right\| \leq \delta_{3} .
$$

Since there exist parameter mismatches between different cells, multicell system (1) cannot be completely synchronized. Instead, we present another type of synchronization, which is defined as follows.

Definition 1. The multicell system (1) is said to reach quasisynchronization or weak synchronization, if, for any initial condition $\left(x_{1}^{\top}(0), \ldots, x_{m}^{\top}(0)\right)^{\top}$, there exists a positive constant $\varepsilon$ such that

$$
\left\|X_{i j}\right\|=\left\|x_{j}-x_{i}\right\| \leq \varepsilon, \quad i, j=1,2, \ldots, N .
$$

The regulatory functions $f_{l}(\cdot)$ and $g_{l}(\cdot)$ are both monotonic increasing functions; there exist two diagonal matrices $K_{1}=\operatorname{diag}\left(k_{11}, k_{12}, \ldots, k_{1 n}\right) \geq 0$ and $K_{1}=$ $\operatorname{diag}\left(k_{21}, k_{22}, \ldots, k_{2 n}\right) \geq 0$ such that the following sector conditions are satisfied:

$$
0 \leq \frac{f_{l}(a)-f_{l}(b)}{a-b} \leq k_{1 l}, \quad 0 \leq \frac{g_{l}(a)-g_{l}(b)}{a-b} \leq k_{2 l},
$$

where $a, b \in R, a \neq b$, and $l=1, \ldots, n$. Notice that the Lur'e system consists of a linear system feedback interconnected with a static nonlinearity $f(\cdot)$ that satisfies a sector condition [20]; multicell system (2) can be regarded as a Lur'e system. Consequently, with help of Lure system method in control theory and Lyapunov direct method, we obtain the following sufficient conditions for quasisynchronization of system (2).

Theorem 2. If there exist symmetric matrices $P>0$ and $\Lambda_{i}=$ $\operatorname{diag}\left(\lambda_{i 1}, \ldots, \lambda_{i n}\right) \geq 0, i=1,2$, and a constant $\rho>0$ such that the symmetric matrix

$$
M=\left[\begin{array}{ccc}
P d+d^{\top} P-2 \eta I_{n}+\rho E & P \alpha+K_{1} \Lambda_{1} & -P \gamma+K_{2} \Lambda_{2} \\
\alpha^{\top} P+K_{1} \Lambda_{1} & -2 \Lambda_{1} & 0 \\
-\gamma^{\top} P+K_{2} \Lambda_{2} & 0 & -2 \Lambda_{2}
\end{array}\right] \leq 0,
$$

where $E \in R^{n \times n}$ is the unit matrix, multicell system (1) is quasisynchronized. The bound on the synchronization errors can be estimated by $\delta / \rho$, where

$$
\delta=\frac{2 \lambda_{\max }(P)}{\lambda_{\min }(P)}\left(\varepsilon_{1} \delta_{1}+\varepsilon_{2} \delta_{2}+\varepsilon_{3} \delta_{3}+\varepsilon_{3}\right) .
$$

Proof. Define a Lyapunov function with respect to multicell system (2) of the following form:

$$
V(x(t))=x^{\top}(t) P x(t),
$$

where $P$ is a positive definite matrix. According to [21], the Lyapunov function is equivalent to the following form:

$$
V(x(t))=\sum_{i=1}^{N} \sum_{j=1}^{N} X_{i j}^{\top} P X_{i j}
$$

where $X_{i j}=x_{j}-x_{i}$ denotes the synchronization errors. 
Based on Lyapunov direct method, if the time derivative of $V(x(t))$ along the trajectories of (2) is negative outside of a neighborhood of the origin $O$, multicell system (2) will achieve quasisynchronization with the errors $X_{i j}$ staying in the neighborhood. Calculating the time derivative of $V(x(t))$ along (2),

$$
\begin{aligned}
\dot{X}_{i j}= & \dot{x}_{j}-\dot{x}_{i} \\
= & \left(d+\delta d_{j}(t)\right) x_{j}+\left(\alpha+\delta \alpha_{j}(t)\right) f\left(x_{j}\right) \\
& -\left(\gamma+\delta \gamma_{j}(t)\right)\left(g\left(x_{j}\right)-e_{n}\right)-\eta I_{n} x_{i} \\
& -\left[\left(d+\delta d_{i}(t)\right) x_{i}+\left(\alpha+\delta \alpha_{i}(t)\right) f\left(x_{i}\right)\right. \\
& \left.\quad-\left(\gamma+\delta \gamma_{i}(t)\right)\left(g\left(x_{i}\right)-e_{n}\right)\right]-\eta I_{n} x_{j} \\
= & \left(d E-\eta I_{n}\right) X_{i j}+\alpha\left(f\left(x_{j}\right)-f\left(x_{i}\right)\right) \\
& -\gamma\left(g\left(x_{j}\right)-g\left(x_{i}\right)\right)+\left(\delta \gamma_{j}(t)-\delta \gamma_{i}(t)\right) I_{n} \\
& +\delta d_{j}(t) x_{j}-\delta d_{i}(t) x_{i}+\delta \alpha_{j}(t) f\left(x_{j}\right) \\
& -\delta \alpha_{i}(t) f\left(x_{i}\right)-\delta \gamma_{j}(t) g\left(x_{j}\right)+\delta \gamma_{i}(t) g\left(x_{i}\right) .
\end{aligned}
$$

We have

$$
\begin{aligned}
\dot{V}=\sum_{i=1}^{N} \sum_{j=1}^{N} X_{i j}^{\top} P & \\
\times[( & \\
& \\
& \left.-\gamma\left(g-\eta I_{n}\right) X_{i j}+\alpha\left(f\left(x_{j}\right)-g\left(x_{i}\right)\right)-f\left(x_{i}\right)\right) \\
& +I_{n}\left(\delta \gamma_{j}(t)-\delta \gamma_{i}(t)\right)+\delta d_{j}(t) x_{j} \\
& -\delta d_{i}(t) x_{i}+\delta \alpha_{j}(t) f\left(x_{j}\right)-\delta \alpha_{i}(t) f\left(x_{i}\right) \\
& \left.-\delta \gamma_{j}(t) g\left(x_{j}\right)+\delta \gamma_{i}(t) g\left(x_{i}\right)\right] .
\end{aligned}
$$

Noticing the sector conditions (7) of $f(\cdot)$ and $g(\cdot)$, we have

$$
\begin{aligned}
& \left(f_{l}\left(x_{j}^{(l)}\right)-f_{l}\left(x_{i}^{(l)}\right)\right)\left(f_{l}\left(x_{j}^{(l)}\right)-f_{l}\left(x_{i}^{(l)}\right)-k_{1 l} X_{i j}^{(l)}\right) \geq 0, \\
& \left(g_{l}\left(x_{j}^{(l)}\right)-g_{l}\left(x_{i}^{(l)}\right)\right)\left(g_{l}\left(x_{j}^{(l)}\right)-g_{l}\left(x_{i}^{(l)}\right)-k_{2 l} X_{i j}^{(l)}\right) \geq 0,
\end{aligned}
$$

where $l=1,2, \ldots, n$. Then, for any $\Lambda_{1}=\operatorname{diag}\left(\lambda_{11}, \ldots, \lambda_{1 n}\right) \geq$ 0 and $\Lambda_{2}=\operatorname{diag}\left(\lambda_{21}, \ldots, \lambda_{2 n}\right) \geq 0$ and any constant $\rho>0$, there holds

$$
\begin{gathered}
\dot{V} \leq \sum_{i=1}^{N} \sum_{j=1}^{N} X_{i j}^{\top} P\left[\left(d E-\eta I_{n}+\rho E\right) X_{i j}\right. \\
\left.+\alpha\left(f\left(x_{j}\right)-f\left(x_{i}\right)\right)\right] \\
-\gamma \sum_{i=1}^{N} \sum_{j=1}^{N} X_{i j}^{\top} P\left(g\left(x_{j}\right)-g\left(x_{i}\right)\right) \\
-2 \sum_{i=1}^{N} \sum_{j=1}^{N} \Lambda_{1}\left(f\left(x_{j}\right)-f\left(x_{i}\right)\right) \\
\times\left(f\left(x_{j}\right)-f\left(x_{i}\right)-K_{1} X_{i j}\right) \\
-2 \sum_{i=1}^{N} \sum_{j=1}^{N} \Lambda_{2}\left(g\left(x_{j}\right)-g\left(x_{i}\right)\right) \\
\times\left(g\left(x_{j}\right)-g\left(x_{i}\right)-K_{2} X_{i j}\right) \\
+\lambda_{\min }(P)\left(\delta\left\|X_{i j}\right\|-\rho\left\|X_{i j}\right\|^{2}\right) \\
=\sum_{i=1}^{N} \sum_{j=1}^{N}\left(\xi_{i j}^{\top} M \xi_{i j}+\lambda_{\min }(P)\left(\delta\left\|X_{i j}\right\|-\rho\left\|X_{i j}\right\|^{2}\right)\right),
\end{gathered}
$$

where $M$ is defined in (8),

$$
\begin{gathered}
\xi_{i j}=\left[\left(x_{j}-x_{i}\right)^{\top},\left(f\left(x_{j}\right)-f\left(x_{i}\right)\right)^{\top},\left(g\left(x_{j}\right)-g\left(x_{i}\right)\right)^{\top}\right]^{\top}, \\
\delta=\frac{2 \lambda_{\max }(P)}{\lambda_{\min }(P)}\left(\varepsilon_{1} \delta_{1}+\varepsilon_{2} \delta_{2}+\varepsilon_{3} \delta_{3}+\varepsilon_{3}\right) .
\end{gathered}
$$

If matrix inequalities (8) and $\left\|x_{j}-x_{i}\right\| \geq \delta / \rho$ hold, one obtains that

$$
\dot{V} \leq \lambda_{\min }(P) \sum_{i=1}^{N} \sum_{j=1}^{N}\left(\delta\left\|X_{i j}\right\|-\rho\left\|X_{i j}\right\|^{2}\right)<0 .
$$

According to Lyapunov direct method, all the trajectories of cells in system (2) will go closer to each other when $X_{i j}$ stay outside of the neighborhood of the origin $O(0, \delta / \rho)$. Therefore, multicell system (2) realizes quasisynchronization with synchronization errors $X_{i j}$ staying in the neighborhood of the origin $O(0, \delta / \rho)$.

Theorem 2 implies that the estimation of the bound on synchronization error is influenced by two factors, the mismatches between cells $\varepsilon_{i}$ and the parameter $\rho$. On one hand, quasisynchronization can be realized if the mismatches are small and the synchronization errors oscillate in a certain neighborhood of the origin. On the other hand, the parameter $\rho$ is determined by the dynamics of the individual cells and reflects the ability of synchronization of the inherent 
dynamics. Furthermore, the larger the parameter $\rho$ is, the smaller the bound on the synchronization errors is.

In fact, the proof of the theorem shows that the mismatches that go against synchronization could be compensated by the linear function $-\rho X_{i j}$. Both the error dynamics caused by the mismatches and $\rho X_{i j}$ could be compensated by the linear function $\Lambda X_{i j}$, which is determined by the diffusion rate of the signals inward the cell membrane $\eta$. Consequently, the parameter $\eta$ measures the quasisynchronization ability of the inherent dynamics of the cells. Therefore, if the two factors mentioned above can be controlled, the synchronization errors can be controlled.

As a special case, if there are no parameter mismatches between different cells, which implies that all the cells are identical, then it is easy to conclude that multicell system (2) realizes complete synchronization. In such a case, multicell systems (1) and (2) could be rewritten as

$$
\begin{gathered}
\dot{a}_{i}=-d_{1 i} a_{i}+\frac{\alpha_{6 i}}{\mu_{6}+C_{i}^{m}}, \quad \dot{A}_{i}=-d_{4 i} A_{i}+\beta_{1 i} a_{i}, \\
\dot{b}_{i}=-d_{2 i} b_{i}+\frac{\alpha_{4 i}}{\mu_{4}+A_{i}^{m}}, \quad \dot{B}_{i}=-d_{5 i} B_{i}+\beta_{2 i} b_{i}, \\
\dot{c}_{i}=-d_{3 i} c_{i}+\frac{\alpha_{5 i}}{\mu_{5}+B_{i}^{m}}+\frac{\alpha_{7 i} S_{i}}{\mu_{7}+S_{i}}, \\
\dot{C}_{i}=-d_{6 i} C_{i}+\beta_{3 i} c_{i}, \\
\dot{S}_{i}=-d_{7 i} S_{i}+\beta_{4 i} A_{i}-\eta\left(S_{i}-S_{e}\right), \\
\dot{S}_{e}=-d_{e} S_{e}+\eta_{e} \sum_{j=1}^{N} \frac{S_{j}-S_{e}}{N}, \\
\dot{x}_{e}=-d_{e} x_{e}+\eta_{e} \sum_{j=1}^{N} \frac{\left(I_{n} x_{j}-x_{e}\right)}{n}, \\
\alpha f\left(x_{i}\right)-\gamma\left(g\left(x_{i}\right)-e_{n}\right)+\eta\left(x_{e}-I_{n} x_{i}\right),
\end{gathered}
$$

where the parameters $d, \gamma, \eta, d_{e}$, and $\eta_{e}$ are defined in multicell system (2). Then we obtain the following corollary.

Corollary 3. If there exist symmetric matrices $P>0$ and $\Lambda_{i}=$ $\operatorname{diag}\left(\lambda_{i 1}, \ldots, \lambda_{i n}\right) \geq 0, i=1,2$, and a constant $\rho>0$ such that the symmetric matrix

$$
M=\left[\begin{array}{ccc}
P d+d^{\top} P-2 \eta I_{n}+\rho E & P \alpha+K_{1} \Lambda_{1} & -P \gamma+K_{2} \Lambda_{2} \\
\alpha^{\top} P+K_{1} \Lambda_{1} & -2 \Lambda_{1} & 0 \\
-\gamma^{\top} P+K_{2} \Lambda_{2} & 0 & -2 \Lambda_{2}
\end{array}\right] \leq 0,
$$

where $E \in R^{n \times n}$ is the unit matrix, multicell system (18) realizes complete synchronization.

\section{Numerical Simulations}

In order to demonstrate the effectiveness of our theoretical analysis, we give numerical examples based on multicell system (1) consisting of 6 cells. Set the parameters as follows:

$$
\begin{gathered}
d_{1}=d_{2}=d_{3}=0.4, \quad d_{4}=d_{5}=d_{6}=0.5, \\
d_{7}=0.016, \quad d_{e}=0.2, \quad \alpha_{4}=\alpha_{5}=\alpha_{6}=1.96, \\
\alpha_{7}=1, \quad \beta_{1}=\beta_{2}=\beta_{3}=0.13, \quad \beta_{4}=0.018, \\
\eta=0.4, \quad \eta_{e}=0.8, \quad \mu_{4}=\mu_{5}=\mu_{6}=\mu_{7}=0.2 .
\end{gathered}
$$

Suppose that the initial mismatches $\delta d_{i}, \delta \alpha_{i}$, and $\delta \beta_{i}$ are taken randomly in the open intervals $\left(-\varepsilon d_{i}, \varepsilon d_{i}\right),\left(-\varepsilon \alpha_{i}, \varepsilon \alpha_{i}\right)$, and $\left(-\varepsilon \beta_{i}, \varepsilon \beta_{i}\right)$, respectively. The evolutions of the synchronization errors $X_{i j}$ are shown to converge to a neighborhood of the origin in Figure 2. As can be seen, the smaller the bound on mismatches between the oscillators is, the smaller the synchronization errors $X_{i j}$ are. And complete synchronization can be realized if $\varepsilon=0$.

In order to verify the relationship between the synchronization errors and the mismatches between cells, the figure of the synchronization errors transition for increasing the mismatches $\varepsilon$ is plotted in Figure 3. It is obvious that quasisynchronization is realized and the synchronization errors increase with the mismatches roughly linearly.

Numerical simulations are also carried out to verify the relationship between the synchronization errors and the diffusion rate of the signals inward the cell membrane in the region $\eta \in[0,2]$. Figure 4 shows that the error bounds depend sensitively on the diffusion rates $\eta$, which play the role of the coupling strength. The increase of the coupling strength makes for the decrease of the synchronization errors, but if the parameter $\eta$ is too large, the dynamics of oscillation of multicell systems (1) breaks. Therefore, all the parameters should be taken appropriately to ensure the dynamics of oscillation.

From Figures 3 and 4, one can see that the error concentrations of gene products are much smaller than those of genes; specifically, the concentrations of biosignals AI remain quasisynchronized even when the mismatches are big. During the time course of achieving synchronization, the synchronous states of AI decrease the mismatches between cells through quorum sensing. And the strong synchronizability of AI makes it act as biosignals to synchronize other chemical molecules. On the other hand, the figures also imply that the error concentrations of genes decrease greatly when the mismatches are decreased. The genes' strong sensitivity to regulations (the synchronous states of the concentrations of gene products, especially AI) is the reason why it can be led to synchronization by AI. The two aspects make up of the mechanisms of collective behavior caused by quorum sensing during transcription, translation, translocation, and signaltransduction.

\section{Conclusions}

Many previous researches studied the collective behavior of biological systems by using complete synchronization. 


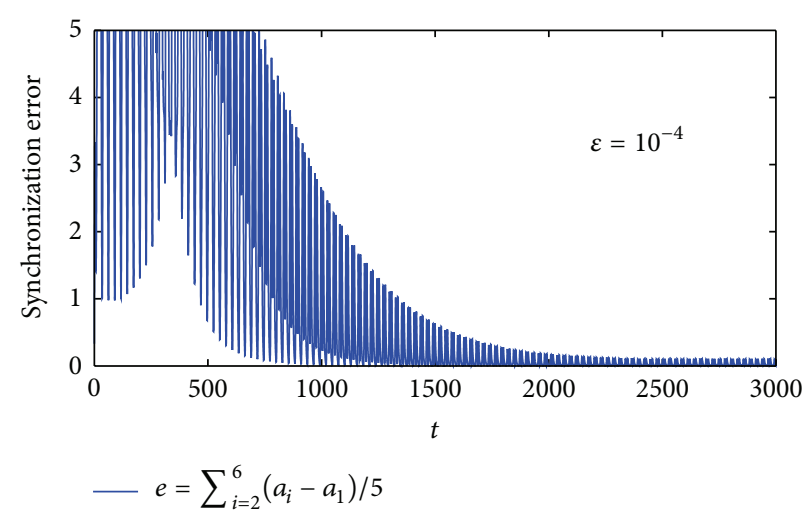

(a)

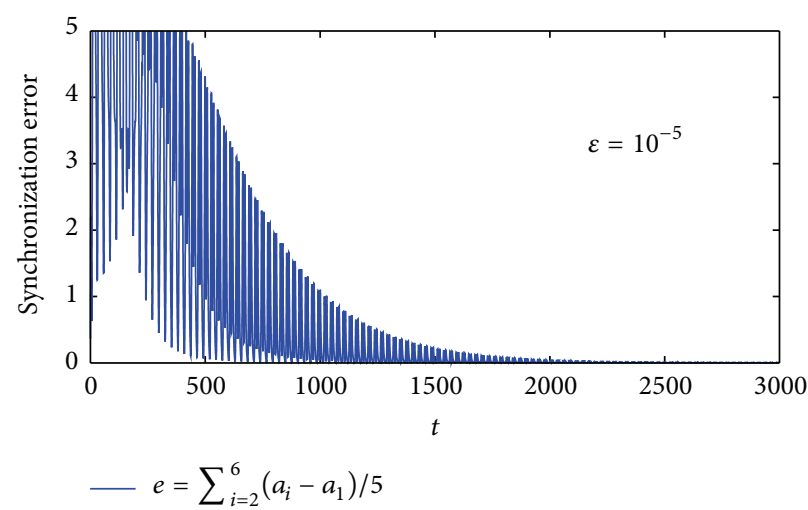

(b)

FIGURE 2: Time evolutions of the synchronization errors $e=\sum_{i=2}^{6}\left(a_{i}-a_{1}\right) / 5$ in multicell system (1), where the parameter $\varepsilon$ is taken as $10^{-4}$ and $10^{-5}$, respectively.

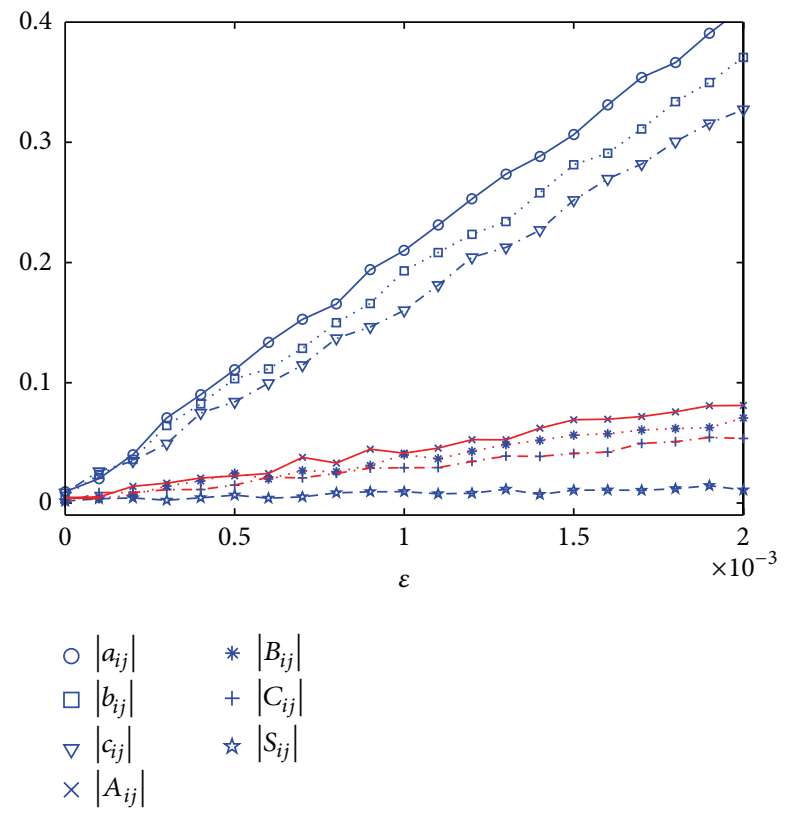

FIGURE 3: Synchronization errors transition for increasing the mismatch bound $\varepsilon \in[0,0.002]$.

However, in many real biological systems, individual organisms are similar but different from each other, and their behaviors are not completely identical either. Therefore, it is meaningful to carry out researches on quasisynchronization instead of complete synchronization. Our results on quasisynchronization in multicell systems coupled by quorum sensing indicate that mismatches between cells can lead to quasisynchronization, and the synchronization errors depend heavily on the parameter mismatches. Theoretical analysis shows that the synchronization errors will decrease if the coupling strength increases. All the results agree well with numerical simulations and biological phenomena in practice.

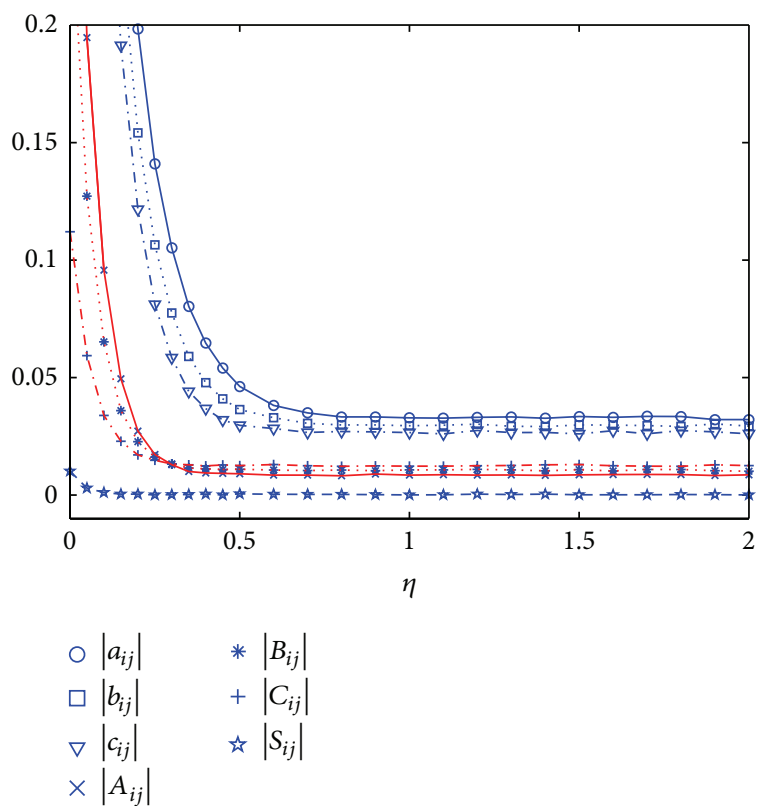

FIGURE 4: Synchronization errors transition for increasing the parameter $\eta \in[0,2]$.

\section{Conflict of Interests}

The authors declare that there is no conflict of interests regarding the publication of this paper.

\section{Acknowledgments}

This research was supported by Zhejiang Provincial NSF of China (nos. LQ12A01003 and LQ12A01002), NNSF of China (nos. 61203155, and 11001069), Guangxi Key Laboratory of Trusted Software (no. kx201417), and the Science Foundation of Guangxi Province (no. 2013GXNSFAA019006). 


\section{References}

[1] R. Femat and J. Alvarez-Ramírez, "Synchronization of a class of strictly different chaotic oscillators," Physics Letters. A, vol. 236, no. 4, pp. 307-313, 1997.

[2] J. A. K. Suykens, P. F. Curran, J. Vandewalle, and L. O. Chua, "Robust nonlinear $H_{\infty}$ synchronization of chaotic Lur' e systems," IEEE Transactions on Circuits and Systems I. Fundamental Theory and Applications, vol. 44, no. 10, pp. 891-904, 1997.

[3] W. Zhang, J. Huang, and P. Wei, "Weak synchronization of chaotic neural networks with parameter mismatch via periodically intermittent control," Applied Mathematical Modelling, vol. 35, no. 2, pp. 612-620, 2011.

[4] X. Liu, T. Chen, J. Cao, and W. Lu, "Dissipativity and quasisynchronization for neural networks with discontinuous activations and parameter mismatches," Neural Networks, vol. 24, no. 10, pp. 1013-1021, 2011.

[5] W. He, F. Qian, Q. Han, and J. Cao, "Lag quasi-synchronization of coupled delayed systems with parameter mismatch," IEEE Transactions on Circuits and Systems I: Regular Papers, vol. 58, no. 6, pp. 1345-1357, 2011.

[6] J. Zhang, Z. Ma, and G. Zhang, "Cluster synchronization induced by one-node clusters in networks with asymmetric negative couplings," Chaos, vol. 23, no. 4, Article ID 043128, 2013.

[7] X. Yang, C. Huang, and J. Cao, "An LMI approach for exponential synchronization of switched stochastic competitive neural networks with mixed delays," Neural Computing and Applications, vol. 21, no. 8, pp. 2033-2047, 2012.

[8] J. Zhang, Z. Ma, and J. Cao, "Full synchronization studied by a set of partitions connected together," Abstract and Applied Analysis, vol. 2013, Article ID 352826, 8 pages, 2013.

[9] M. B. Elowitz and S. Leibier, "A synthetic oscillatory network of transcriptional regulators," Nature, vol. 403, no. 6767, pp. 335$338,2000$.

[10] R. Wang and L. Chen, "Synchronizing genetic oscillators by signaling molecules," Journal of Biological Rhythms, vol. 20, no. 3, pp. 257-269, 2005.

[11] J. Zamora-Munt, C. Masoller, J. Garcia-Ojalvo, and R. Roy, "Crowd synchrony and quorum sensing in delay-coupled lasers," Physical Review Letters, vol. 105, no. 26, Article ID 264101, 2010.

[12] A. F. Taylor, M. R. Tinsley, F. Wang, Z. Huang, and K. Showalter, "Dynamical quorum sensing and synchronization in large populations of chemical oscillators," Science, vol. 323, no. 5914, pp. 614-617, 2009.

[13] D. J. Schwab, A. Baetica, and P. Mehta, "Dynamical quorumsensing in oscillators coupled through an external medium," Physica D: Nonlinear Phenomena, vol. 241, no. 21, pp. 1782-1788, 2012.

[14] D. McMillen, N. Kopell, J. Hasty, and J. J. Collins, "Synchronizing genetic relaxation oscillators by intercell signaling," Proceedings of the National Academy of Sciences of the United States of America, vol. 99, no. 2, pp. 679-684, 2002.

[15] J. Garcia-Ojalvo, M. B. Elowitz, and S. H. Strogatz, "Modeling a synthetic multicellular clock: repressilators coupled by quorum sensing," Proceedings of the National Academy of Sciences of the United States of America, vol. 101, no. 30, pp. 10955-10960, 2004.

[16] S. P. Boyd, L. E. I. Ghaoui, E. Feron, and V. BalaKrishnan, "Linear matrix inequalities in system and control theory," SIAM Studies in Applied Mathematics, vol. 15, pp. 119-126, 1994.
[17] C. Huang, H. Kuang, X. Chen et al., "An LMI approach for dynamics of switched cellular neural networks with mixed delays," Abstract and Applied Analysis, vol. 2013, Article ID 870486, 8 pages, 2013.

[18] C. Li, L. Chen, and K. Aihara, "Synchronization of coupled nonidentical genetic oscillators," Physical Biology, vol. 3, no. 1, pp. 37-44, 2006.

[19] C. Huang and J. Cao, "Convergence dynamics of stochastic Cohen-Grossberg neural networks with unbounded distributed delays," IEEE Transactions on Neural Networks, vol. 22, no. 4, pp. 561-572, 2011.

[20] M. Vidyasagar, Nonlinear Systems Analysis, Prentice-Hall, Englewood Cliffs, NJ, USA, 2nd edition, 1993.

[21] C. W. Wu, Synchronization in Coupled Chaotic Circuits and Systems, World Scientific, Singapore, 2002. 


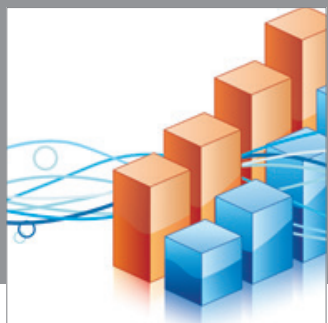

Advances in

Operations Research

mansans

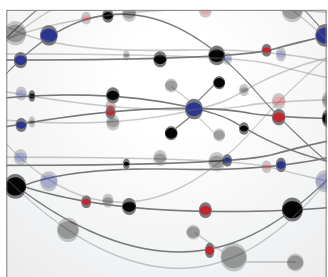

The Scientific World Journal
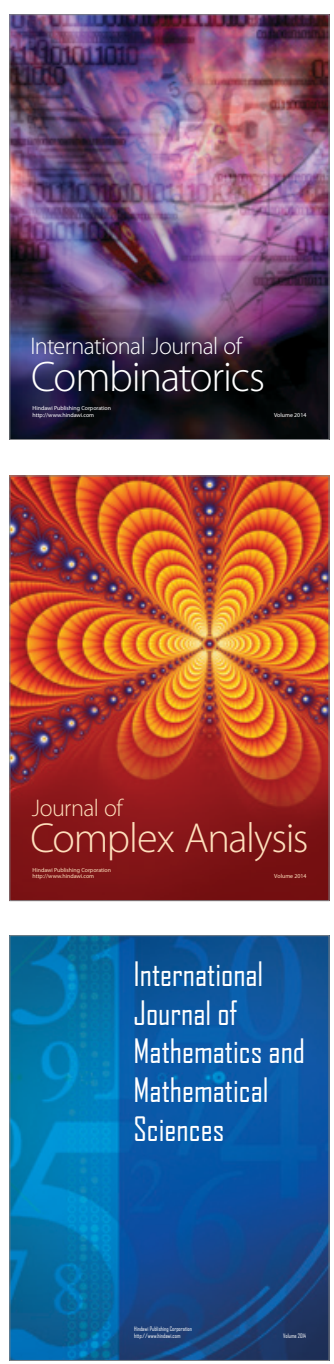
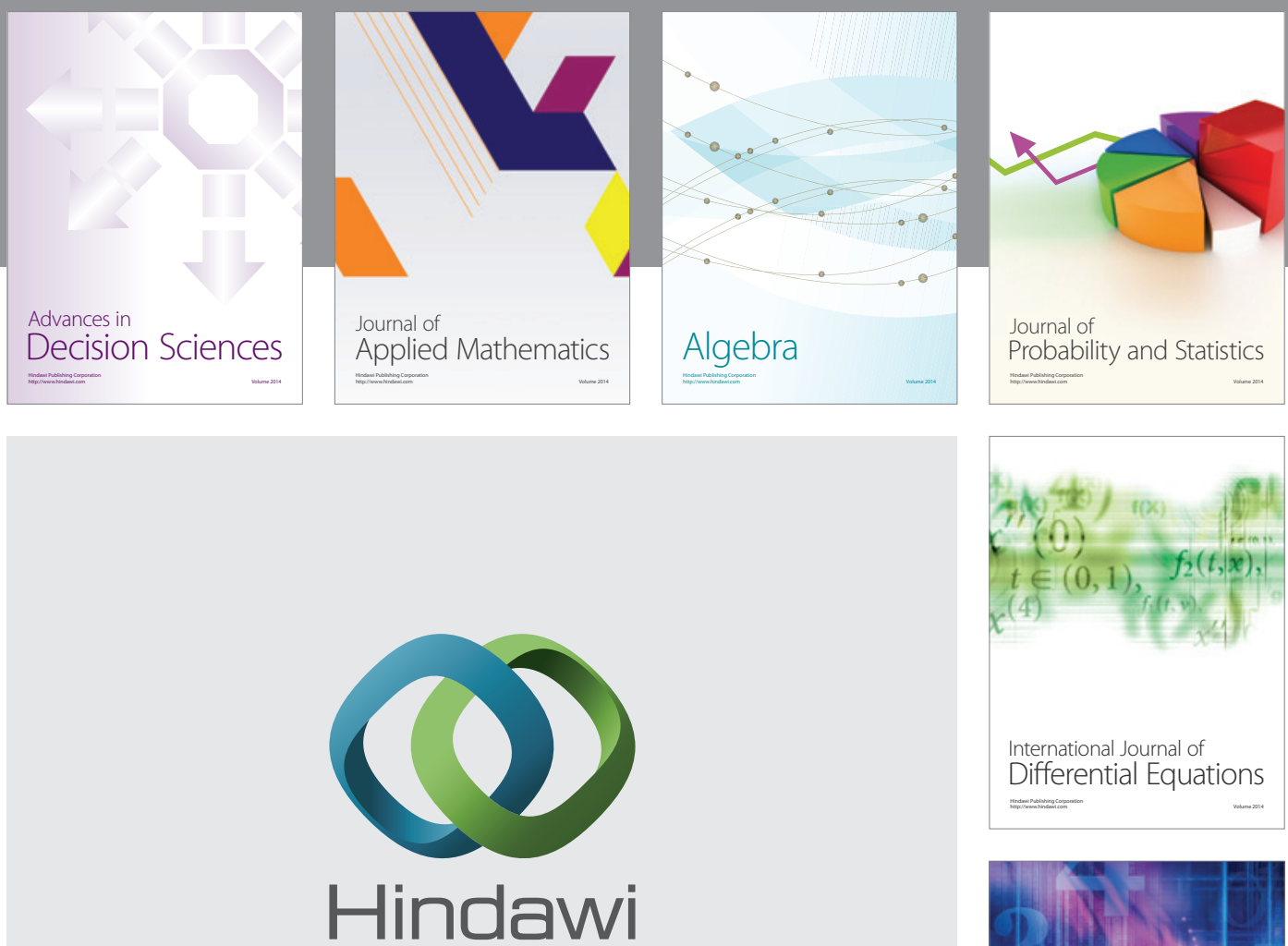

Submit your manuscripts at http://www.hindawi.com
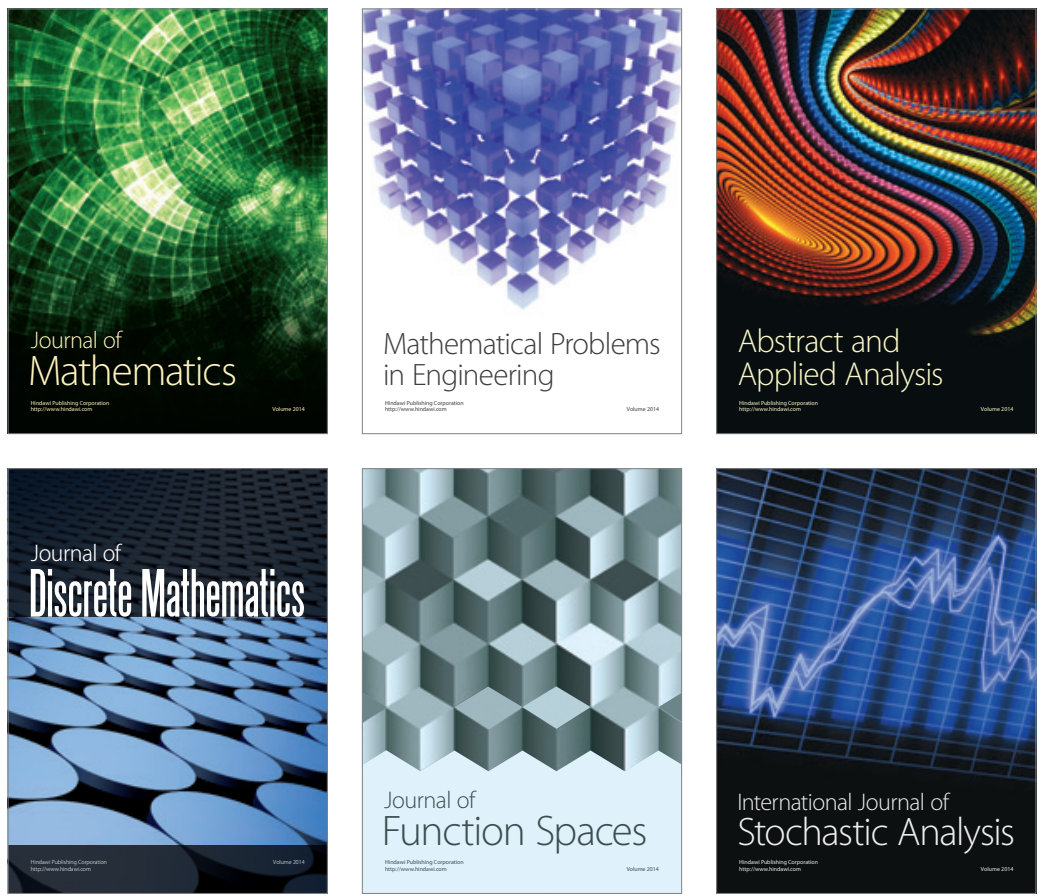

Journal of

Function Spaces

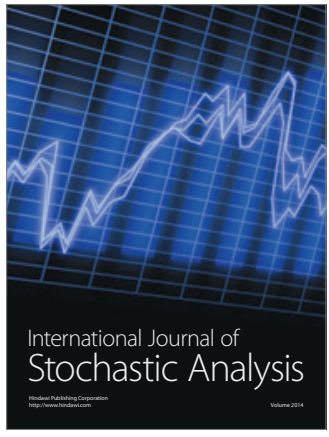

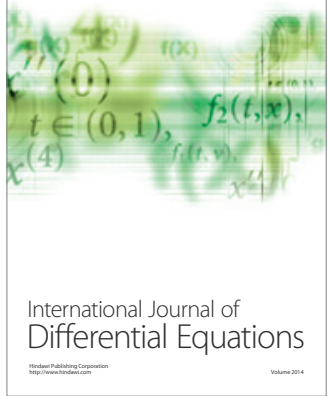
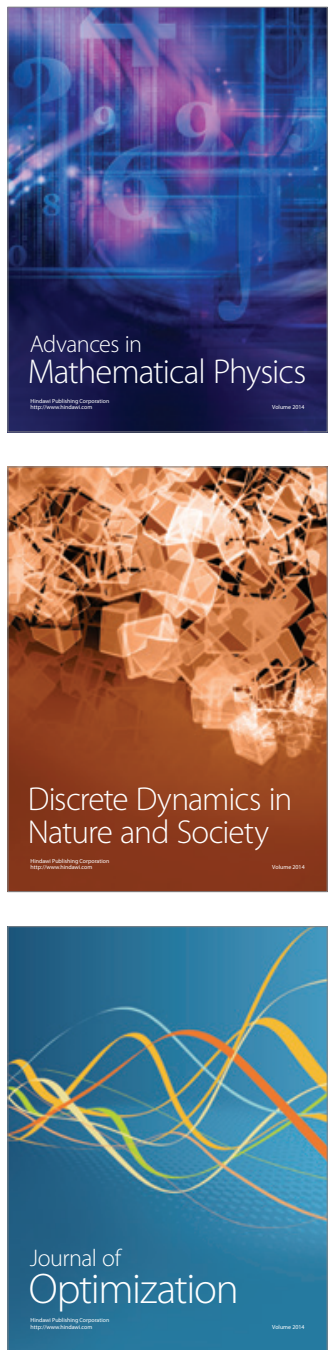